SILVA, C.R.A. e SILVA, F.L. Radiodiagnóstico intervencionista. PUBVET, Londrina, V. 5, N. 33, Ed. 180, Art. 1217, 2011.

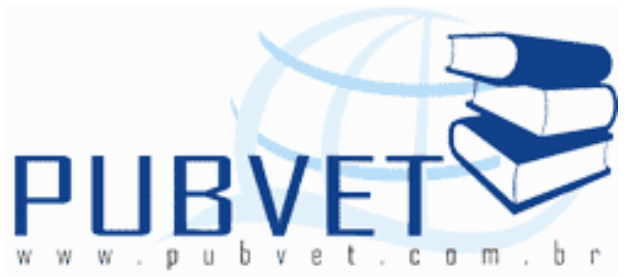

PUBVET, Publicações em Medicina Veterinária e Zootecnia.

\title{
Radiodiagnóstico intervencionista
}

Catarina Rafaela Alves da Silva ${ }^{1}$ e Francisco Lima Silva ${ }^{2}$

${ }^{1}$ Doutoranda em Ciência Animal - UFPI

${ }^{2}$ Professor Doutor Universidade Federal do Piauí

\section{Resumo}

Todas as intervenções médicas que utilizam a radiação $X$ para obter um diagnóstico, são chamados de radiodiagnóstico. O radiodiagnóstico é a prática na qual um feixe de radiação é usado para produzir uma imagem com o propósito de se obter um diagnóstico que irá excluir ou avaliar o desenvolvimento de uma condição patológica. A radiologia intervencional é uma prática em que imagens são geradas por meio do uso de radiações, sendo os equipamentos de fluoroscopia considerados fontes desta radiação. Constantemente a tecnologia avança para alcançar cada vez mais um impacto na capacidade de percepção e de exibição da informação diagnóstica e com isso conta com apoio da ultrassonografia intervencional bastante utilizada em procedimentos biópsias e drenagens. Esta revisão de literatura teve como objetivo adquirir informações sobre radiodiagnóstico e sua importância, assim como o conhecimento das técnicas utilizadas para radiodiagnóstico intervencional. 
SILVA, C.R.A. e SILVA, F.L. Radiodiagnóstico intervencionista. PUBVET, Londrina, V. 5, N. 33, Ed. 180, Art. 1217, 2011.

\title{
Interventional radiodiagnosis
}

\begin{abstract}
All medical procedures that use X-rays for a diagnostic radiology are called. The radiology is the practice in which a beam of radiation is used to produce an image with the purpose of obtaining a diagnosis that will exclude or assess the development of a pathological condition. Interventional Radiology is a practice in which images are generated through the use of radiation, and the fluoroscopy equipment considered sources of radiation. Constantly advancing technology to achieve a more impact on the ability of perception and display of diagnostic information and thus has the support of interventional ultrasound procedures in very used in biopsies and drainages. This literature review aimed to acquire information about a diagnostic radiology and its importance, as well as knowledge of techniques used in interventional radiology.
\end{abstract}

\section{INTRODUÇÃO}

O radiodiagnóstico intervencionista é defendido por procedimentos que compreendem de intervenções diagnósticas e terapêuticas guiadas por acesso percutâneo ou outros, normalmente realizados sob anestesia local ou sedação (CANEVARO, 2009), no qual se faz uso de radiação X (MIRANDA, 2009). A especialidade médica que estuda a radiologia possui várias áreas de aplicação, como pode ser visto na Figura 1. 
SILVA, C.R.A. e SILVA, F.L. Radiodiagnóstico intervencionista. PUBVET, Londrina, V. 5, N. 33, Ed. 180, Art. 1217, 2011.

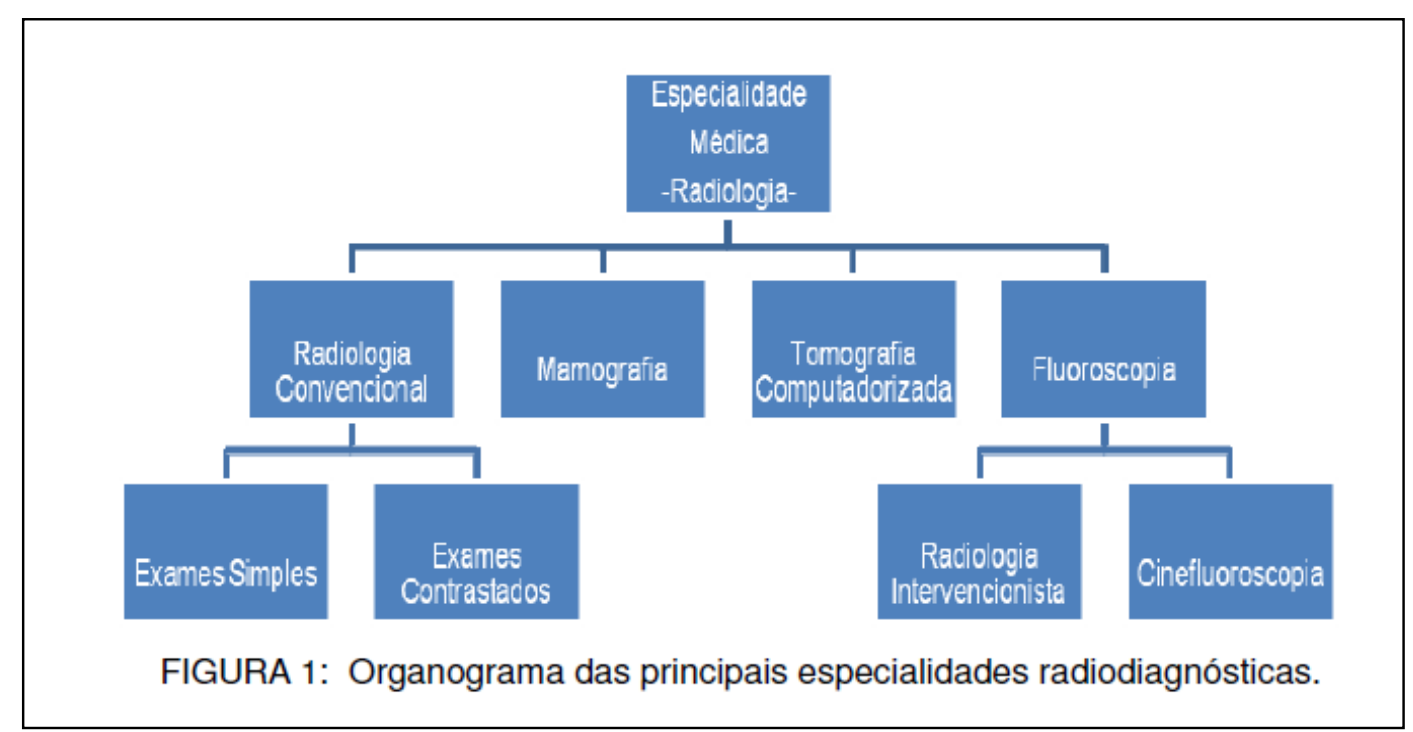

(MIRANDA, 2009)

Pela utilização da radiologia, radioscopia, ecocardiografia, tomografia computadorizada e/ou ressonância magnética, como guia, inserem-se agulhas, cateteres e tubos em qualquer parte do organismo (TURRINI, 2010).

A radiologia convencional pode utilizar uma técnica simples ou contrastada. A mamografia é um radiodiagnóstico específico para mamas e utiliza técnicas, sistema de radiação, material do anodo e as qualidades do feixe diferentes da convencional. Já a tomografia computadorizada possui um aparelho que utiliza vários sensores e feixes estreitíssimos onde é possível a visualização da imagem radiografada por planos transversais e reconstruções desses planos. E por fim a fluoroscopia utiliza-se por feixes contínuos, no qual se objetiva obter uma imagem em tempo real, podendo ser gravadas e avaliadas posteriormente.

Além destas técnicas que utilizam radiação eletromagnética, existem outros exames que não utilizam os raios $X$, mas são tratadas dentro $d a$ especialidade radiológica como a ressonância nuclear magnética e ultrassom (MIRANDA, 2009). 
SILVA, C.R.A. e SILVA, F.L. Radiodiagnóstico intervencionista. PUBVET, Londrina, V. 5, N. 33, Ed. 180, Art. 1217, 2011.

\section{RADIOLOGIA INTERVENCIONISTA}

Pode ser definida como a área de atuação médica que emprega o uso das radiações ionizantes (SOUZA; SOARES, 2008).

A grande vantagem desta técnica é a possibilidade da realização de procedimentos complexos com cortes cirúrgicos de pequena extensão, a diminuição da probabilidade de infecções, rápido restabelecimento do paciente, a redução do tempo de internação e a diminuição dos custos hospitalares, tratando-se de uma técnica minimamente invasiva, segura e altamente eficaz (CANEVARO, 2009). A radiologia intervencional oferece uma alternativa para o tratamento cirúrgico de muitas condições e pode eliminar a necessidade de hospitalização, em alguns casos (MIRANDA, 2009). Pode ser utilizada em diversos casos médicos como mostrado na tabela 1 :

TABELA 1: Técnicas de Diagnósticos e tratamentos utilizando a radiologia intervencional.

\begin{tabular}{|c|c|}
\hline Técnica & Procedimento \\
\hline Angiografia & $\begin{array}{l}\text { Radiografia das artérias e veias para detectar obstruçōes e } \\
\text { estreitamento das mesmas. A radiologia intervencional utiliza cateteres } \\
\text { e guias para a desobstrução com a colocação de stent ou insuflando } \\
\text { um pequeno balāo. }\end{array}$ \\
\hline Angioplastia & Desobstrução da veia através da insuflação de um pequeno balāo \\
\hline Embolizaçāo & $\begin{array}{l}\text { É a inserçāo de um cateter com substâncias coagulantes no interior } \\
\text { dos vasos sangüíneos para estancar uma hemorragia. }\end{array}$ \\
\hline Gastrectomia & $\begin{array}{l}\text { Inserção de um cateter especial para alimentação do paciente, } \\
\text { quando ele é impossibilitado de se alimentar pela boca. }\end{array}$ \\
\hline $\begin{array}{l}\text { Ultra-som } \\
\text { intravascular }\end{array}$ & Auxilio com guia na ultra-sonografia intravenoso \\
\hline $\begin{array}{l}\text { Colocação de } \\
\text { stent }\end{array}$ & $\begin{array}{l}\text { O stent é uma pequena mola de metal, inserida e guiada para dentro } \\
\text { dos vasos sanguíneos, recuperando o lúmen do vaso e garantindo } \\
\text { maior fluidez do sangue. }\end{array}$ \\
\hline $\begin{array}{l}\text { Injeçōes de } \\
\text { anticoagulantes }\end{array}$ & Inserçōes de substâncias anticoagulantes \\
\hline $\begin{array}{l}\text { Colocação de } \\
\text { filtros sangüíneos }\end{array}$ & $\begin{array}{l}\text { Inserçōes de pequenos filtros para evitar pequenos coágulos nos } \\
\text { vasos sangüíneos. }\end{array}$ \\
\hline $\begin{array}{l}\text { Inserçōes de } \\
\text { cateteres }\end{array}$ & $\begin{array}{l}\text { Inserçōes de cateteres para levar medicamentos em tratamentos de } \\
\text { tumores malignos e nos casos de transplantes de medula e } \\
\text { hemodiálises }\end{array}$ \\
\hline
\end{tabular}

(MIRANDA, 2009) 
SILVA, C.R.A. e SILVA, F.L. Radiodiagnóstico intervencionista. PUBVET, Londrina, V. 5, N. 33, Ed. 180, Art. 1217, 2011.

Com o aumento das técnicas e da complexidade dos procedimentos em radiodiagnóstico e com o aparecimento de lesões radioinduzidas, e devido ao fato que a maioria destes profissionais teve pouca ou nenhuma formação em proteção radiológica, existe uma necessidade urgente de informá-los sobre os riscos da radiação e como minimizá-los na sua prática quotidiana (LUZ, 2004).

\subsection{Efeitos estocásticos e determinísticos na radiologia intervencionista}

As altas doses ministradas nos procedimentos intervencionistas, se não conhecidas e otimizadas, podem aumentar os riscos de efeitos estocásticos e ocasionar efeitos determinísticos em pacientes e profissionais, como pode ser visto na figura 2, (CANEVARO, 2009).

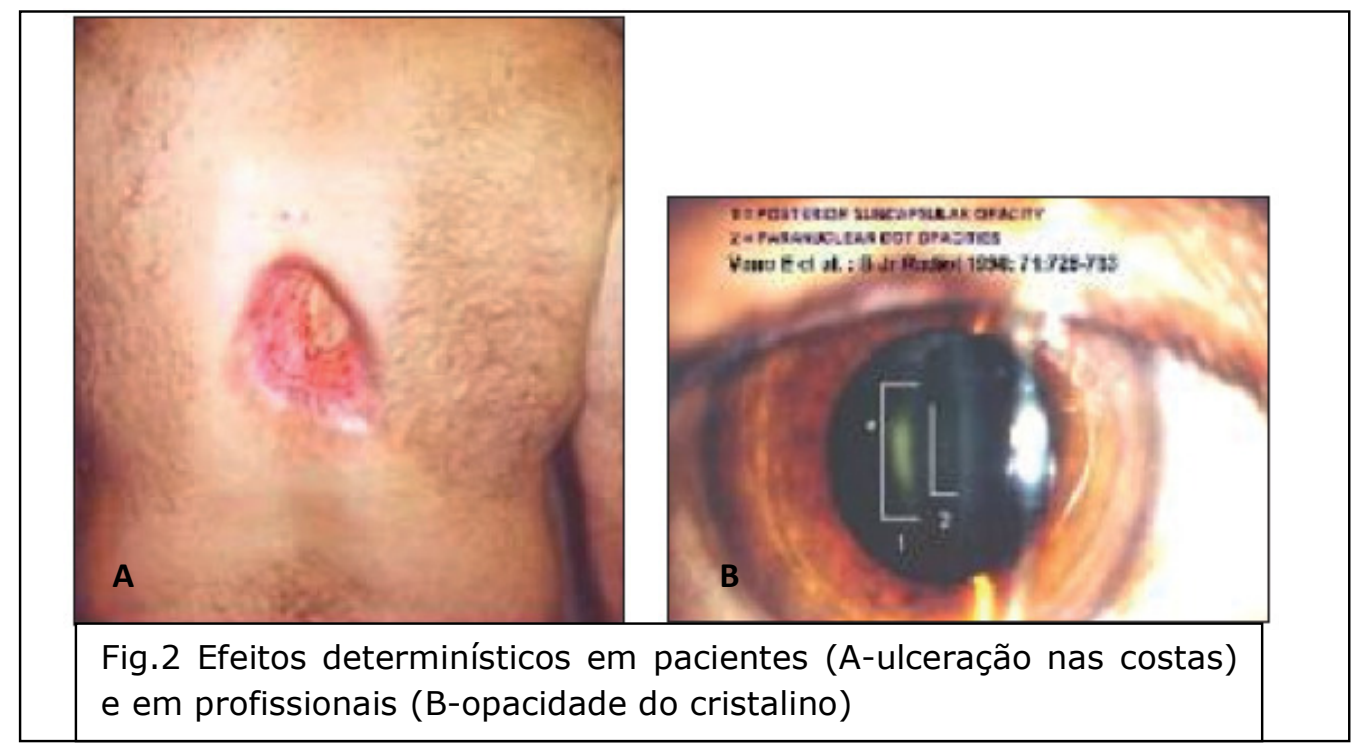

(CANEVARO, 2009)

Estas lesões não aparecem imediatamente, pelo que o médico não pode perceber danos ao observar o paciente imediatamente após o exame. Além das lesões em pacientes, têm sido observados casos de catarata e sérios danos nas mãos dos médicos que realizam os exames. $O$ risco de aparecimento de lesões na pele está associado por tempos prolongados de fluoroscopia. A 
SILVA, C.R.A. e SILVA, F.L. Radiodiagnóstico intervencionista. PUBVET, Londrina, V. 5, N. 33, Ed. 180, Art. 1217, 2011.

maioria das lesões severas pode ser evitada conhecendo e quantificando não apenas a radiação que sai do tubo de raios $X$ e chega ao paciente, mas também a qualidade da imagem suficiente para a intervenção. Além disso, é indispensável fazer uso racional dos raios X (CANEVARO, 2009).

\subsection{Sistema de radioproteção}

A proteção radiológica tem por objetivo proteger adequadamente os profissionais sem limitar desnecessariamente as práticas benéficas que utilizam com a radiação ionizante (SOUZA; SOARES, 2008). No caso dos profissionais, o uso de ferramentas de radioproteção e a aplicação de boas práticas podem diminuir os valores de dose efetiva assim como o risco de lesões no cristalino e na pele (CANEVARO, 2009).

Conforme regulamentações da Associação Brasileira de Normas Técnicas (ABNT), todo profissional que trabalha com radiodiagnóstico deve usar um dosímetro sempre e somente quando estiver na área de risco e ainda submetêlo mensalmente para a leitura dos dados nele contido, a fim de monitorizar a radiação individual acumulada, fornecendo, assim, informações acerca da exposição à radiação ionizante (SOUZA; SOARES, 2008).

O uso de equipamentos de proteção é indispensável. O uso de aventais de chumbo pode reduzir a $5 \%$ a dose dos profissionais na sala do exame, e devem ser usados por toda a equipe. Blindagens especiais devem ser colocadas entre o paciente e o médico. Para evitar o surgimento de catarata, os médicos devem usar óculos plumbíferos (CANEVARO, 2009). As blindagens são utilizadas para deter a propagação das radiações, evitando, assim, exposições desnecessárias de pessoas envolvidas com equipamentos que emitem radiação ionizante. Sua eficiência é determinada pela capacidade de penetração dos raios $X$, assim como pela natureza e espessura do material de blindagem (SOUZA; SOARES, 2008). 
SILVA, C.R.A. e SILVA, F.L. Radiodiagnóstico intervencionista. PUBVET, Londrina, V. 5, N. 33, Ed. 180, Art. 1217, 2011.

As luvas cirúrgicas plumbíferas, que são comercializadas, possuem um fator de atenuação contra a radiação que varia de 5 a $20 \%$, dependendo do modelo. Porém, mesmo com as luvas protetoras, o médico deve evitar a exposição de sua mão no campo de irradiação ou sob o intensificador de imagem, pois esse procedimento raramente se constitui em uma necessidade médica. Por diminuir a sensação tátil, poder prolongar o procedimento e também causar maior radiação na mão, visto que essas luvas não dão proteção eficaz, o seu uso é pouco difundido (BALTER, 1999).

Os profissionais devem seguir um padrão de proteção radiológica que tem como princípios fundamentais os seguintes pontos: a) justificação qualquer atividade envolvendo a radiação ou exposição deve ser justificada em relação a alternativas disponíveis e também produzir um benefício significativo para a sociedade. $O$ beneficio tem de ser tal que compense o procedimento ou exame radiológico; b) otimização - sempre utilizar a menor dose possível de radiação; c) limitação da dose - as doses individuais de profissionais e de indivíduos do público não devem ultrapassar os limites primários de doses anuais que constam em normas da CNEN, as quais retratam normas estabelecidas por órgãos internacionais de proteção radiológica; d) prevenção - todo esforço deve ser direcionado para estabelecer medidas rígidas para a prevenção de acidentes (SOUZA; SOARES, 2008).

\section{ULTRASSONOGRAFIA INTERVENCIONISTA}

A tecnologia continua a ter um grande impacto na capacidade de percepção e de exibição da informação diagnóstica. Apesar de todos os avanços na ultrassonografia na última década, nas áreas tradicionais de contraste, resoluções espacial e temporal, índice de interferência, artefatos e estética de imagem, grandes avanços tecnológicos continuam. Dentre os exames que estão envolvidos na ultrassonografia intervencional estão: drenagem percutânea de lesões cavitárias, pielografia percutânea, biópsias 
SILVA, C.R.A. e SILVA, F.L. Radiodiagnóstico intervencionista. PUBVET, Londrina, V. 5, N. 33, Ed. 180, Art. 1217, 2011.

aspirativas e colecistocentese percutânea guiada por ultrassom (MATTOON et al., 2004).

\subsection{Ultrassonografia intra-operatória}

A ultrassonografia intra-operatória é realizada com o uso de transdutores pequenos, específicos e com estrutura linear de alta resolução, disponíveis em vários formatos para adaptarem-se melhor ao órgão ou ao procedimento de interesse. Esta orientação por ultrassom foi usada para auxiliar vários procedimentos cirúrgicos, para determinar margens de lesões e o grau de ressecamento das lesões, para detectar lesões parenquimatosas profundas não identificadas no pré-operatório (MATTOON et al., 2004).

Outra contribuição desse método é a sua capacidade de identificar, no campo operatório, a posição do ducto de Wirsung com relação às lesões pancreáticas. Isto permite que os tumores neuroendócrinos pouco agressivos (epecialmente os insulinomas) e tumores císticos serosos (adenoma microcístico) sejam removidos por enucleação, em vez de ressecções pancreáticas, sem que haja lesão do ducto pancreático principal. Este fato auxilia na escolha da melhor opção terapêutica a ser usada (MACHADO et al., 2002).

\subsection{Endossonografia}

A ultrassonografia endoscópica refere-se ao uso de um pequeno transdutor de alta freqüência incorporado à ponta de um endoscópio, no qual é introduzido dentro da cavidade corpórea por via oral, transrretal ou transvaginal. A endossonografia ainda não é considerada um método de diagnóstico de rotina na medicina humana, mas fornece informações complementares àquelas obtidas pela endoscopia convencional e pela ultrassonografia transcutânea (MATTOON et al., 2004). 
SILVA, C.R.A. e SILVA, F.L. Radiodiagnóstico intervencionista. PUBVET, Londrina, V. 5, N. 33, Ed. 180, Art. 1217, 2011.

\subsection{Laparoscopia ultrassonográfica}

Refere-se à inserção de um transdutor ultrassonográfico fixo ou flexível atreves de um acesso laparoscópico para obter uma imagem direta de estruturas intrabdominais ou intratorácicas (MACHI, 1999). A Laparoscopia ultrassonográfica pode fornecer informação importante para o cirurgião quanto ao planejamento do procedimento cirúrgico ou à otimização do manejo medicamentoso do caso (MATTOON et al., 2004).

\subsection{Ultrassonografia endovascular}

Os transdutores ultrassonográficos podem agora ser pequenos ao ponto de serem incorporados dentro de um cateter fino e introduzidos pela via intravenosa em lugares específicos (LIU; GOLDBERG, 1999). As áreas mais promissoras da aplicação clínica são quantificação do grau de estenose arterial e a monitorização dos efeitos da angioplastia nas artérias coronárias e periféricas. Procedimentos intervencionais, tais como a colocação de uma prótese endovascular de stent e a localização dos cateteres de ablação de miocárdio, são possíveis (MATTOON et al., 2004).

É um procedimento importante para o uso endovascular de stents nas doenças de aorta torácica descendente está recebendo uma atenção crescente, uma vez que é um procedimento promissor, menos invasivo e boa alternativa para a abertura cirúrgica (SOARES et al.,2009).

\subsection{Biomicroscopia ultrassonográfica}

A Biomiosopia ultrassonográfica alcança uma resolução extraordinária pelo uso de transdutores de ultra-alta-frequência. Foi relatada primeiramente em 1990 sendo utilizada principalmente para o estudo do olho. Este transdutores ultra-alta-frequência continuarão a ser desenvolvidos e seu uso 
SILVA, C.R.A. e SILVA, F.L. Radiodiagnóstico intervencionista. PUBVET, Londrina, V. 5, N. 33, Ed. 180, Art. 1217, 2011.

irá se expandir para outras áreas, tais como a avaliação de nervos, ligamentos, tendões e vascularização periférica (MATTOON et al., 2004).

\section{ULTRASSONOGRAFIA TRIDIMENSIONAL}

Com os avanços no processamento de dados e na capacidade de memória, atualmente estão disponíveis transdutores bidimensionais capazes de expandir o campo de visão para a terceira dimensão. Esta imagem 3-D é constituída pelo uso de um avançado programa de computador com os dados de volume adquiridos em séries de planos no modo-B, que são tanto paralelos entre si como separados por diferenciais angulares regulares (MATTOON et al., 2004).

Vários estudos estão sendo realizados para se avaliar a aplicabilidade clínica da ultrassonografia tridimensional e um deles está inserido o exame obstétrico podendo facilitar a deteç̧ão de algumas anormalidades fetais como algumas anormalidades morfológicas de membros, estruturas ósseas, face e orelhas que podem não ser identificadas pela ultrassonografia bidimensional (HASE, 2002).

\section{CONSIDERAÇÕES FINAIS}

A utilização do radiodiagnóstico intervencionista é de grande importância por possibilitar alternativas para o diagnóstico de doenças não detectáveis ou difíceis de detectar nos procedimentos de imagens convencionais como, por exemplo, a radiologia convencional que utiliza radiografias simples e contrastadas. A radiologia intervencional utiliza-se por acesso percutâneo para a realização do exame possibilitando maior acurácia nas imagens. A ultrassonografia intervencional é um procedimento que complementa todo o radiodiagnóstico intervencionista por meio de realizações de procedimentos guiados pelo ultrassom. Devido a todos estes benefícios na detecção de imagens com mais qualidade é importante ressaltar o uso dos sistemas de 
SILVA, C.R.A. e SILVA, F.L. Radiodiagnóstico intervencionista. PUBVET, Londrina, V. 5, N. 33, Ed. 180, Art. 1217, 2011.

proteção contra radiação utilizada para estes exames com a finalidade de não trazer problemas futuros para a saúde do profissional envolvido.

\section{REFERÊNCIAS BIBLIOGRÁFICAS}

CANEVARO, L. Aspectos físicos e técnicos da radiologia intervencionista. Revista Brasileira de Física Médica. v.3,n.1,p.101-115,2009.

BALTER, S. Radiation safety in the cardiac catheterization laboratory: operational radiation safety. Catheter Cardiovasc Interv. v.47, p.347-53, 1999.

HASE, E.A. Aplicabilidade da ultra-sonograifia tridimensional em obstetrícia. Rev. Assoc. Med. Bras. v.48, n.3, 2002.

LIU, J.B; GOLDBERG, B.B. 2-D and 3-D endoluminal ultrassond: vascular and nonvascular applications. Ultrassond Med Biol. v.25, p.159-173, 1999.

LUZ, E. S. Proteção radiológica em radiologia intervencionista: estudo de caso [dissertação]. Rio de Janeiro: Instituto Militar de Engenharia; 2004

MACHI, J. Intraoperative and laparoscopic ultrasond. Surg. Oncol. Clín. Noth. Am. v.8, p.205-226, 1999.

MACHADO, M.M.; ROSA,A.C.F.;MARCEL,W.B.;MACHADO,C.C.;CERRI,G.G. Ultra-sonografia intra-operatória (USIO) do pâncreas e das vias biliares. Rad. Bras. São Paulo. v.35, n.3, p.105-108, 2002.

MATTOON,J.S.;PENNINCK,D.G.;WISNER,E.R.;NYLAND,T.G.;AULD,D.M. Técnicas avançadas e tendências futuras. In: NYLAND,T.G.;MATTON,J.S. Ultra-som diagnóstico em pequenos animais. São Paulo: Roca,2004. Cap.19.p.439-454.

MIRANDA, J.A. Desenvolvimento de uma metodologia para a calibração de instrumentos de medida utilizadas no controle de qualidade em radiodiagnóstico intervencional. São Paulo. 105f. Dissertação (Tecnologia nuclear). Instituo de Pesquisas Energéticas e Nucleares. São Paulo, 2009.

SOARES, J.S.; SOARES,L.C.; PENA,F.M.; AREAS,G.S.; CUNHA,C.C. Reparo endovascular da ruptura de aneurisma de aorta torácica descendente: Relato de Caso. Rev. SOCERJ. v. 22 ,n.2, p.108-111, 2009.

SOUZA, E.; SOARES,J.P.M. Correlações técnicas e ocupacionais da radiologia intervencionista. J. Vasc. Bras. v.7, n.4, p.341-350, 2008.

TURRINI, R.N.T. Unidades de radiologia intervencionista/hemodinâmica: caracterização de enfermeiro e da estrutura da unidade. Rev. Eletr. Enf. v.12, n.2, p.315-320, 2010. 